\section{Our panel of experts highlight the most important research articles across the spectrum of topics relevant to the field of neurodegenerative disease management}

Expert panel: Donald Grosset, Southern General Hospital, UK; Dag Aarsland Stravanger University Hospital, Norway; Aleksey Kansantsev, Harvard Medical School, MA, USA

Cummings JL, Henchcliffe C, Schaier S, Simuni T, Waxman A, Kemp P. The role of dopaminergic imaging in patients with symptoms of dopaminergic system neurodegeneration. Brain doi: 10.1093/brain/awr177 (2011) (Epub ahead of print).

The selective degeneration of dopaminergic neurons of the midbrain is a pathological process strongly associated with Parkinson's disease (PD). However, confident clinical diagnosis of a specific syndrome in patients presenting with symptoms of parkinsonism can be challenging, particularly in the early stages of the disease, owing to overlap in signs and symptoms. Given that early intervention may lead to superior longterm outcomes, accurate early diagnosis is a pressing requirement. Biomarkers such as dopamine system imaging may be useful in this regard, and it has been suggested that functional imaging with specific dopaminerelated tracers may enable assessment of synaptic function or detection of alterations in neurotransmitter levels.

In this article, Cummings and colleagues review the evidence supporting the utility of imaging of the dopamine transporter using SPECT for diagnosis of neurodegenerative disorders. They explain that dopamine transporter imaging can indicate the presence of underlying nigrostriatal neurodegeneration, and thus can

enable differentiation between PD and nondegenerative disorders such as essential tremor, vascular parkinsonism and druginduced parkinsonism, in addition to differentiating between dementia with lewy bodies/PD with dementia and Alzheimer's disease (AD). In patients with clinically inconclusive parkinsonism, imaging has the potential to provide important further information to aid diagnosis. Moreover, by allowing these etioliogical distinctions, imaging may identify patient groups in whom dopaminergic therapy may be beneficial or, conversely, require a different therapeutic approach. However, the authors conclude that dopamine transporter imaging is not efficacious in differentiating between PD and atypical parkinsonism (e.g., multiple system atrophy, corticobasal degeneration and progressive supranuclear palsy); they suggest that postsynaptic dopaminergic imaging may be helpful in this situation, but more data are required for a definitive conclusion.

Finally, the authors speculate on further, potential uses of dopamine transporter imaging, which include identification of patients with premotor Parkinson's disease, monitoring disease progression during tests of novel therapeutics, and as an inclusion criterion for clinical trials.

- Written by Peter Livermore. Article suggested by Donald Grosset.
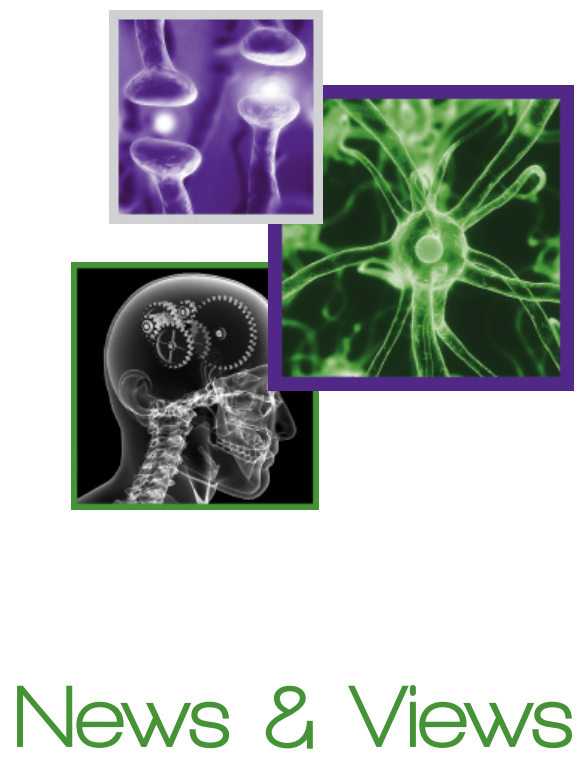

News

Journal Watch 
Schillaci O, Chiaravalloti A,

Pierantozzi $M$ et al. Different patterns

of nigrostriatal degeneration in tremor

type versus the akinetic-rigid and

mixed types of Parkinson's disease at

the early stages: molecular imaging

with 123I-FP-CIT SPECT. Int. J. Mol.

Med. doi: 10.3892/ijmm.2011.764

(2011) (Epub ahead of print).

Parkinson's disease is a syndrome associated with significant phenotypic heterogeneity owing to various associations of motor and nonmotor symptoms, the onset of motor complications, the cognitive disorder's appearance and various other factors. However, the importance of discriminating between different forms of PD could aid in understanding the pathophysiology of extrapyramidal signs - various motor disorders such as acute dystonic reactions, pseudoparkinsonism, or akathisia, resulting from therapeutic administration of dopamine antagonists.

Here, Schillaci et al. aimed to investigate the relationship between the clinical motor phenotypes of PD and the scintigraphic pattern of 123I-FP-CIT SPECT. The authors examined 47 patients with early idiopathic PD (25 males; 22 females; mean age $58 \pm 2$ years) and subdivided them into different groups of clinical forms based on dominance of resting tremor $(\mathrm{n}=20)$, bradykinesia plus rigidity $(\mathrm{n}=20)$ and the presence of both clinical signs (mixed type; $\mathrm{n}=7$ ). Comparing this status with the semiquantitative analysis of 123I-FP-CIT SPECT, they found that tremor type patients showed a lower reduction of 123I-FP-CIT uptake than akinetic-rigid-type patients in contralateral caudate and putamen nuclei. Tremortype patients exhibited greater uptake of 123I-FP-CIT in the ipsilateral caudate and putamen than akinetic-rigid-type patients. Finally, significant differences in the striatal uptake between tremor type and akinetic-rigid type patients were found only in the ipsilateral and contralateral caudate.

The results suggest that akinetic-rigid patients have greater dopaminergic system involvement than tremor type patients; a difference that is present from the initial stage of the disease. In addition, this study indicates that PD phenotypes might be related to other systems besides dopaminergic involvement.

- Written by Peter Livermore. Article suggested by Donald Grosset.

Zwilling D, Huang SY,

Sathyasaikumar KV et al. Kynurenine

3-monooxygenase inhibition in blood ameliorates neurodegeneration. Cell 145(6), 863-874 (2011);

Campesan S, Green EW, Breda C et al. The kynurenine pathway modulates neurodegeneration in a Drosophila model of Huntington's disease. Curr. Biol. 21(11), 961-966 (2011).

Huntington's disease (HD) neurodegeneration is strongly associated with a polyglutamine-expanded huntingtin gene (HTT), which is transcribed into mutant Huntingtin protein $(\mathrm{mHtt})$. This protein is toxic to certain cells, causing mainly striatal damage but also affecting other regions of the brain. However, the pathophysiological mechanisms of HD are still not completely understood. Recently, studies have implicated the kynurenine pathway (KP) in HD pathology, and have indicated that metabolites in the KP generated by tryptophan degradation may play a causative role in this disease. This has been largely owing to the fact that glutamate receptor-mediated excitotoxicity and the generation of free radicals have been correlated with decreased levels of the neuroprotective metabolite kynurenic acid.

In these two recent studies, a collaborative effort led by researchers at Gladstone Institute of Neurological Disease, University of California, CA, USA, and the Department of Genetics, University of Leicester, UK, who previously identified a potential role of the KP pathway in HD, has sought to further investigate the role of this pathway in the etiopathogenesis of HD. The first of the two studies employed a transgenic Drosophila melanogaster model of HD to investigate the therapeutic effects of KP manipulation. The authors found that genetic and pharmacological inhibition of kynurenine 3-monooxygenase (KMO) increased levels of the neuroprotective metabolite kynurenic acid (KYNA) relative to the neurotoxic metabolite 3-hydroxykynurenine and prevents neurodegeneration. In addition, genetic knockout of tryptophan 2,3-dioxygenase, which provides the first and rate-limiting step in the KP pathway, showed a similar neuroprotective shift in the direction of KYNA synthesis. Supplementation of KYNA and 3-hydroxykynurenine to the HD-model flies directly affected neurodegeneration, which emphasized the role of these metabolites.

In the second study, published in Cell, the researchers synthesized and characterized JM6, a small-molecule prodrug inhibitor of KMO. Chronic oral administration of JM6 in mice demonstrated that the prodrug can inhibit KMO in the blood, increasing KYNA levels and reducing extracellular glutamate in the brain. Moreover, JM6 prevented deficits in spatial memory, anxiety-related behavior and synaptic loss in a transgenic AD mouse model. In a HD mouse model, JM6 extended the life-span of the mice, in addition to reducing synaptic loss and decreasing microglial activation.

Both studies underscore the importance of the KP pathway in neurodegeneration and together suggest several potential targets for therapeutic intervention in diseases such as HD.

- Written by Peter Livermore. Article suggested by Aleksey Kazantsev.

Banerjee S, Hellier J, Dewey M et al. Sertraline or mirtazapine for depression in dementia (HTA-SADD): a randomised, multicentre, double-blind, placebo-controlled trial. Lancet 378(9789), 403-411 (2011).

Depression is common in $\mathrm{AD}$ and other dementias, and has important clinical implications such as reduced quality of life and excess disability. Despite this, there is little evidence on which to choose management strategies for people with dementia and depression. 
There have been few studies of antidepressants, with small sample sizes and inconsistent results.

In the HTA-SADD trial, Banerjee and coworkers aimed to conduct the definite study to address this important question. In this pragmatic, multicenter, randomized, placebo-controlled study, treatment with sertraline $(150 \mathrm{mg} /$ day $)$ and mirtazapine (45 mg/day) was compared with placebo after 13 and 39 weeks. With 326 patients enrolled with clinically significant depression, recruited from old-age psychiatry services in UK, this was the largest study in this population with a selective serotonin re-uptake inhibitor. The authors found that all three treatment groups improved significantly during the first 13 weeks, and the improvement was maintained at week 39. However, there was no significant differences between the groups on the primary (Cornell scale for depression in dementia) or any of the secondary outcome measures such as quality of life and cognition or carer burden. Similar findings emerged when subgroup analyses were performed in patients with high or moderate depression severity. By contrast, adverse events were more common in the two treatment groups. The authors discuss the possible role for people with dementia and depression of standard low-intensity care delivered by the old-age psychiatry team, and suggest that the routine use of antidepressants for people with dementia and depression should be reconsidered.

\section{- Article suggested and written by} Dag Aarsland.

\section{Financial \& competing interests disclosure}

The authors have no relevant affiliations or financial involvement with any organization or entity with a financial interest in or financial conflict with the subject matter or materials discussed in the manuscript. This includes employment, consultancies, honoraria, stock ownership or options, expert testimony, grants or patents received or pending, or royalties. No writing assistance was utilized in the production of this manuscript. 